\title{
PPInfer: a Bioconductor package for inferring functionally related proteins using protein interaction networks [version
}

\section{1; peer review: 1 approved with reservations]}

\section{Dongmin Jung(i)1, Xijin Ge (iD)2}

${ }^{1}$ Avison Biomedical Research Center, Yonsei University, Seoul, South Korea

${ }^{2}$ Department of Mathematics and Statistics, South Dakota State University, Brookings, SD, USA

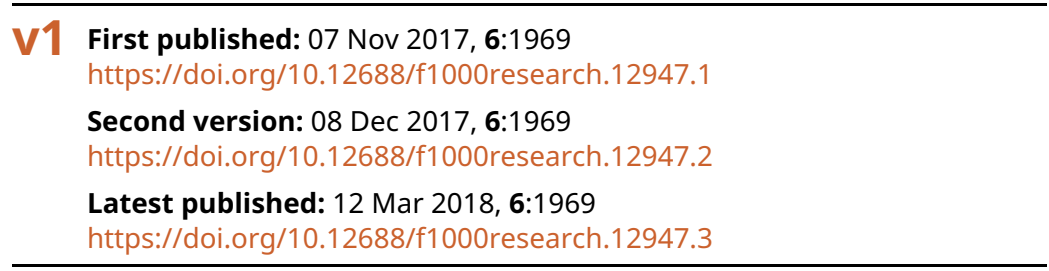

\section{Abstract}

Interactions between proteins occur in many, if not most, biological processes. This fact has motivated the development of a variety of experimental methods for the identification of protein-protein interaction (PPI) networks. Leveraging PPI data available STRING database, we use network-based statistical learning methods to infer the putative functions of proteins from the known functions of neighboring proteins on a PPI network. This package identifies such proteins often involved in the same or similar biological functions. The package is freely available at the Bioconductor web site (http://bioconductor.org/packages/PPInfer/).

\section{Keywords}

graph mining, protein-protein interaction, pseudo-absence, semisupervised learning, support vector machine

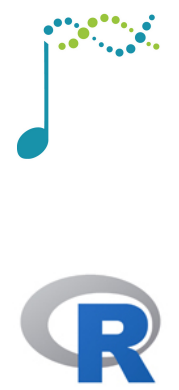

This article is included in the Bioconductor gateway.

This article is included in the RPackage gateway.

Open Peer Review
$\begin{aligned} & \text { Approval Status } \\ & \end{aligned}$
$\begin{aligned} & \text { version 3 } \\ & \text { (revision) }\end{aligned}$
12 Mar 2018
version 2
(revision)
08 Dec 2017
version 1

1. Cathy H. Wu, University of Delaware, Newark, USA

Karen E. Ross, Georgetown University Medical Center, Washington, USA

2. Helen V. Cook ID, University of Copenhagen, Copenhagen, Denmark Any reports and responses or comments on the article can be found at the end of the article. 
This article is included in the Interactive Figures

collection.

This article is included in the Machine learning:

life sciences collection.

Corresponding author: Xijin Ge (gexijin@gmail.com)

Author roles: Jung D: Conceptualization, Investigation, Methodology, Software, Visualization, Writing - Original Draft Preparation; Ge X: Conceptualization, Funding Acquisition, Investigation, Methodology, Validation, Visualization, Writing - Review \& Editing

Competing interests: No competing interests were disclosed.

Grant information: This material is based upon work supported by the National Science Foundation/EPSCoR Grant Number IIA-1355423 and by the State of South Dakota

The funders had no role in study design, data collection and analysis, decision to publish, or preparation of the manuscript.

Copyright: @ 2017 Jung D and Ge X. This is an open access article distributed under the terms of the Creative Commons Attribution License, which permits unrestricted use, distribution, and reproduction in any medium, provided the original work is properly cited.

How to cite this article: Jung $D$ and Ge X. PPInfer: a Bioconductor package for inferring functionally related proteins using protein interaction networks [version 1; peer review: 1 approved with reservations] F1000Research 2017, 6:1969

https://doi.org/10.12688/f1000research.12947.1

First published: 07 Nov 2017, 6:1969 https://doi.org/10.12688/f1000research.12947.1 


\section{Introduction}

The functions of many proteins remain unknown. This is a big challenge in functional genomics. As proteins that consist of the same protein complex are often involved in the same cellular process, the pattern of protein-protein interactions (PPIs) can give information regarding protein function. Thus PPI databases can be useful to predict protein function, complementing conventional approaches based on protein sequence analyses.

Many databases store information on protein-protein interactions as well as protein complexes. For example, the Biological General Repository for Interaction Datasets (BioGRID) includes over 500,000 manually annotated interactions ${ }^{1}$. The STRING database aims to provide a critical assessment and integration of protein-protein interactions, including direct (physical) as well as indirect (functional) associations. The basic interaction unit in STRING is the functional association, i.e. a specific and productive functional relationship between two proteins ${ }^{2}$.

Once the PPI has been obtained experimentally, there are numerous methods to analyze the network. A neighbor counting method for protein function prediction was developed ${ }^{3,4}$. The theory of Markov random fields was used to infer functions using protein-protein interaction data and the functional annotations of its interaction protein partners $^{5}$. There are many algorithms that try to integrate multiple sources of data to infer functions ${ }^{6-8}$. We propose a method that can infer the putative functions of proteins by solving classification problems and thus identifying closely connected proteins known to be involved in a certain process.

We use the kernel method for the graph as a similarity measure between proteins, instead of using the first or second level neighbors, and thus our proposed method provides scores or distances derived from a graph kernel. The main advantage of the proposed method is that the neighbors of a set of proteins can be ranked in terms of scores. Generally, we need two or more classes for classification problem. Although we only know the target, we want to apply semi-supervised learning techniques to the PPI through this package. Thus, the main idea of this method is how to find another class so that two classes can be used for classification problem. Eventually, we can classify proteins to identify such closely related proteins by using this package. Finally, functional enrichment analyses such as ORA and GSEA are incorporated to predict the protein function from the closely related proteins.

\section{Methods}

The support vector machine (SVM) is one of most widely used methods for classification ${ }^{9}$. Suppose we have a dataset in the real space and that each point in our dataset has a corresponding class label. A SVM is involved in a convex optimization problem to separate data points in the dataset according to their class, by maximizing distance between class and minimizing a penalty for misclassification for each class, at the same time. Unfortunately, the graph data is not in the real space. Cover's theorem ${ }^{10}$ provides the useful idea behind a nonlinear SVM, which is to find an optimal separating hyperplane in high-dimensional feature space mapped by using a suitable kernel function, just as we did for the linear SVM in original space.

Graph (network) data is ubiquitous and graph mining tries to extract novel and insightful information from data. Graph kernels are defined in the form of kernel matrices, based on the normalized Laplacian matrix for a graph. The best-known kernel in a graph is the diffusion kernel ${ }^{11}$. The motivation is that it is often easier to describe the local neighborhood than to describe the structure of the whole space ${ }^{12}$. Another method is called a regularized Laplacian matrix $^{13}$ and is widely used in areas such as spectral graph theory, where properties of graphs are studied in terms of their eigenvalues and vectors of adjacency matrices ${ }^{14}$. Broadly speaking, kernels can be thought of as functions that produce similarity matrices ${ }^{15}$. In the package, we choose only the regularized Laplacian matrix as a graph kernel for the PPI.

In many biological problems, datasets are often compounded by imbalanced class distribution, known as the imbalanced data problem, in which the size of one class is significantly larger than that of the other class. Many classification algorithms such as a SVM are sensitive to data with an imbalanced class problem, leading to a suboptimal classification. It is desirable to compensate the imbalance effect in model training for more accurate classification. A possible solution to this problem is to use the one-class SVM (OCSVM) by learning from the target class only ${ }^{16}$. In one-class classification, it is assumed that only information of one of the classes, the target class, is available, and no information is available from the other class, known as the background. The OCSVM can be solely applied because we have only one class, the target. However, it is known that one-class classifiers seldom outperform two-class classifiers when the data from two class are available ${ }^{17}$.

The strategy of this package is to make use of the OCSVM and classical SVM, sequentially. First, we apply the OCSVM by training a one-class classifier using the data from the known class only. Let $n$ be the number of proteins in the 
target class. This model is used to identify distantly related proteins among remaining $N-n$ proteins in the background. Indeed, we do not know whether or not each of proteins in the background interacts with the proteins of the target. Thus, it does not always imply that proteins in the background do not interact with the target. In fact, their associations with the target are not observed to date yet. Proteins with zero similarity with the target class are extracted. Then they are potentially defined as the other class by pseudo-absence selection methods ${ }^{18}$ from spatial statistics. The target class can be seen as real presence data. The main idea of the proposed method is to adopt the pseudo-absence class. For the data to be balanced, assume that two classes contain the same number of proteins. Next, by the classical SVM, these two classes are used to identify closely related proteins whose scores ranging from -1 to 1 are close to 1 among remaining $N-2 n$ proteins. Semi-supervised learning can be applied to make use of large unlabeled data and small labeled data. Some of these methods directly try to label the unlabeled data. Eventually, those found by this procedure can be functionally linked to the target proteins. This is usually based on the assumption that unannotated proteins have similar functions as their interacting proteins.

\section{Use cases}

We need a list of proteins and the kernel matrix to infer functionally related proteins. With the STRING database for mouse, it is supposed that the target is the set of proteins in the Ras signaling pathway from the KEGG pathway (http://www.genome.jp/kegg-bin/show_pathway?mmu04014).

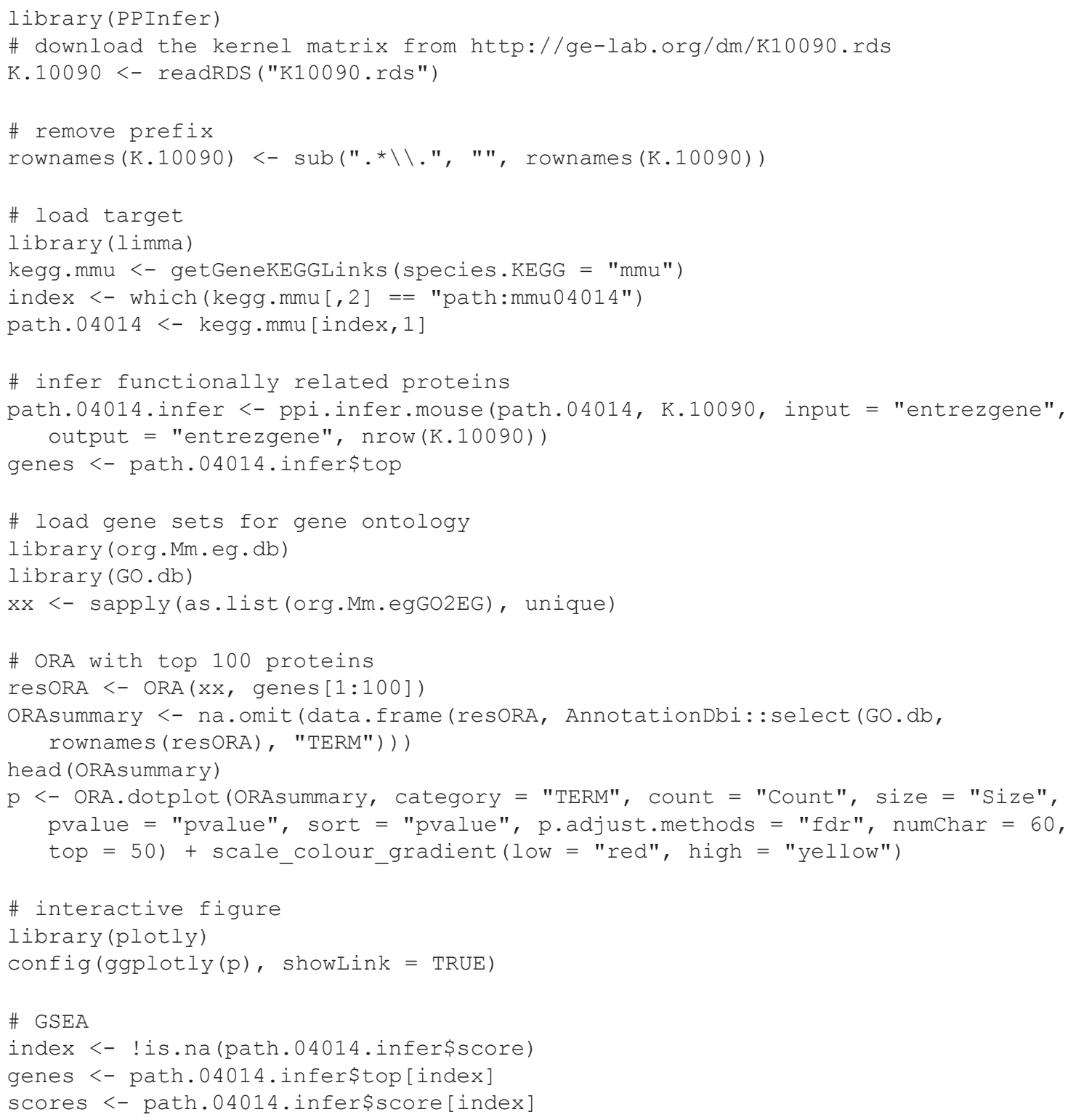




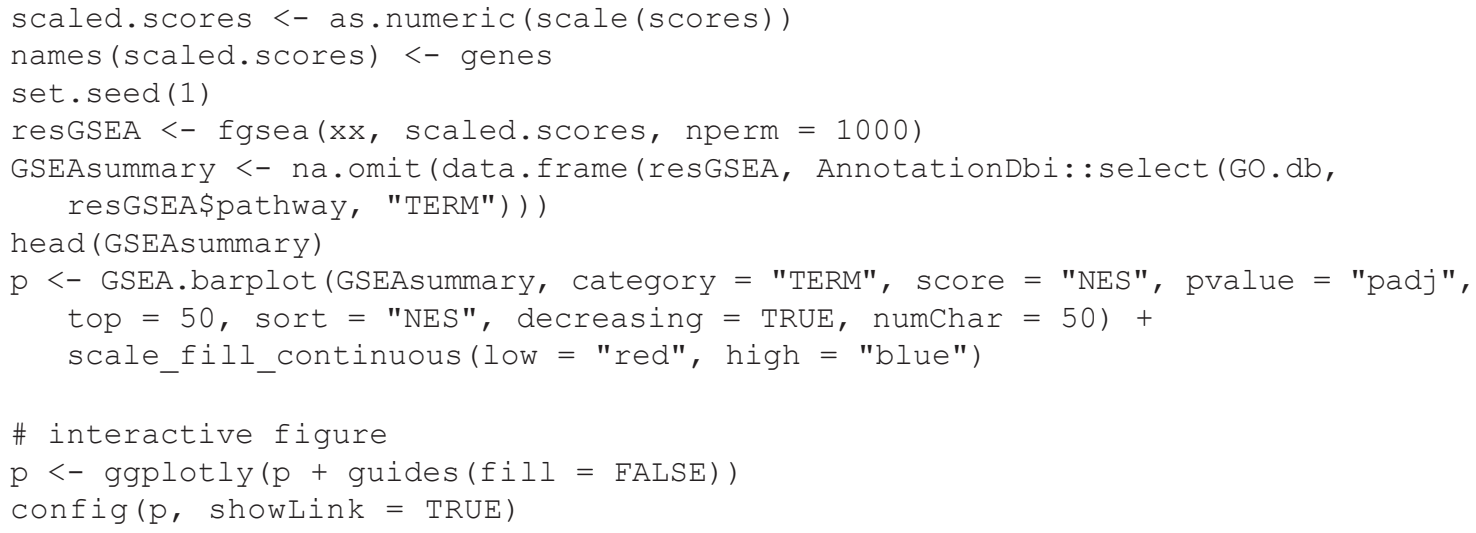

The tyrosine kinase receptor binds to a ligand in the extracellular space. Then its cytoplasmic domain undergoes conformational change that forms dimerization, resulting in transphophotyrosine. The $\mathrm{SH} 2$ domain recognizes this phosphotyrosine. The Grb2 adaptor protein containing SH2 binds these receptors and recruits Sos, which is the Ras-GEF inducing Ras, to release its GDP and bind a GTP instead. The Ras protein is activated by this guanine nucleotide exchange factor. GTP-activated Ras can activate PI3K. Once PIP3 is formed by PI3K, an Akt/PKB kinase can become tethered via its $\mathrm{PH}$ domain. Once activated, Akt/PKB proceeds to phosphorylate a series of protein substrates, leading to aiding survival by reducing the possibility of apoptotic suicide program. For this reason, several GO terms about the RTK and PI3K pathways are shown in Figure 1. We can find cell migration due to the integrin and FAK. MAP kinase activity, which is a downstream of Ras signaling and causes cell proliferation, is statistically significant.

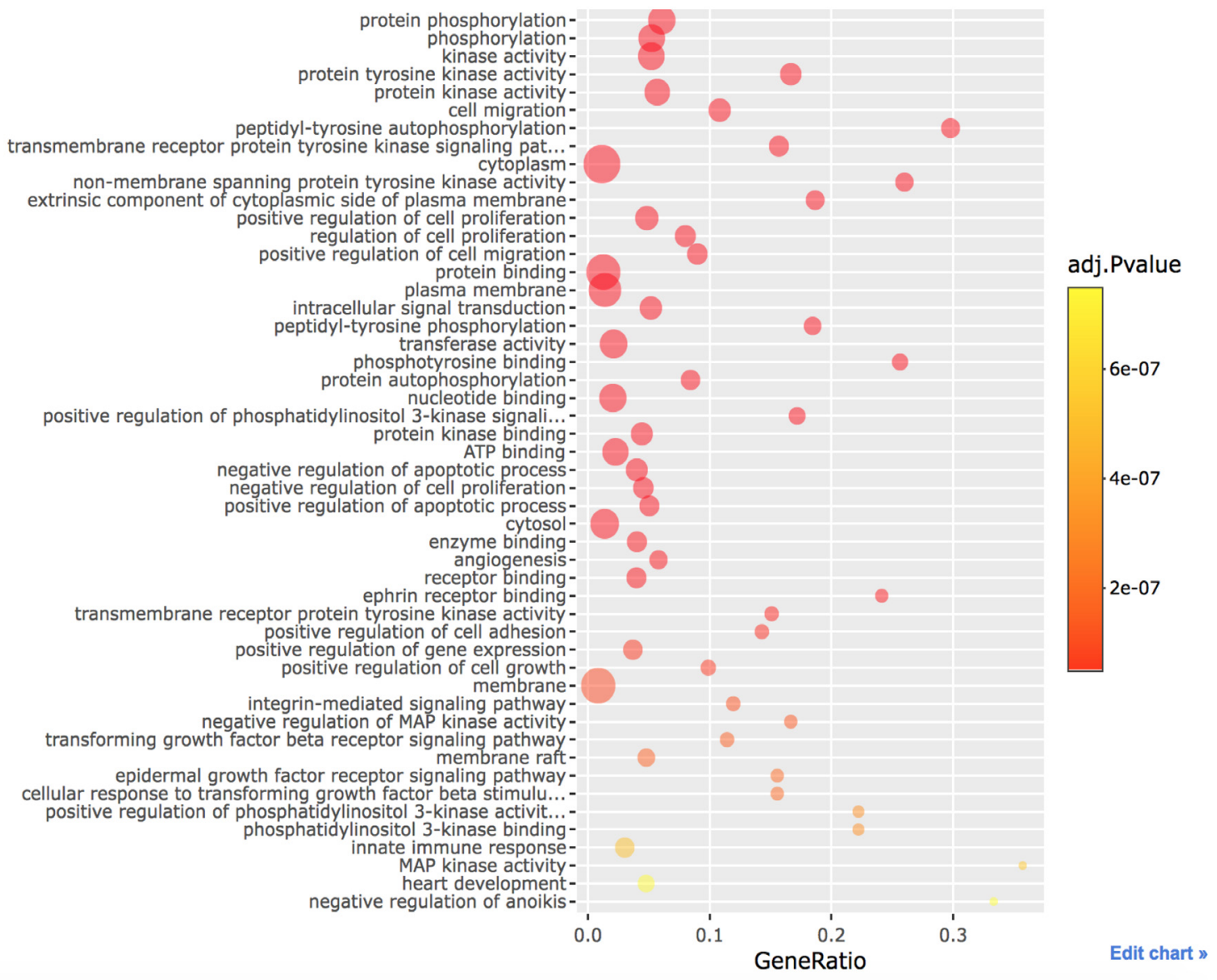

Figure 1. Top 50 categories and their adjusted p-values from the ORA with gene ontology, based on 100 proteins most closely related to the target, Ras signaling pathway. This figure was generated with Plotly (https://plot.ly/). 
In the GSEA, the gene list is sorted by the standardized score showing how much they are functionally close to the target. Genes with higher scores are functionally more related to the target. The positive values of the enrichment score indicate enrichment at the top of the ranked gene list. Proteins that are closely related to the target are enriched in categories with high enrichment scores. In other words, these proteins are depleted in categories with low enrichment scores. We can see the RTK, MAPK and Wnt signaling pathways in Figure 2. One possible reason is that Akt can activate $\beta$-catenin both indirectly and indirectly.

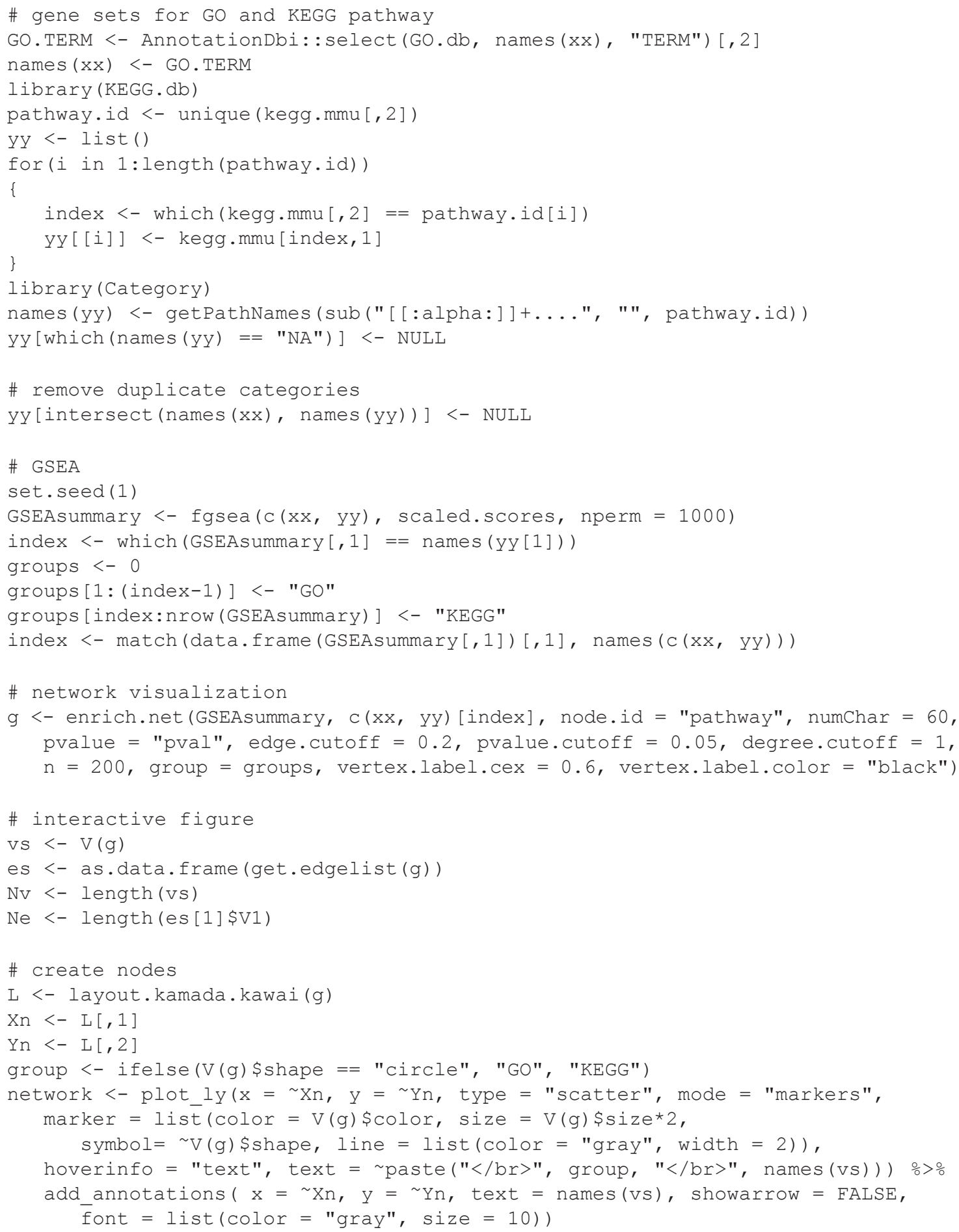




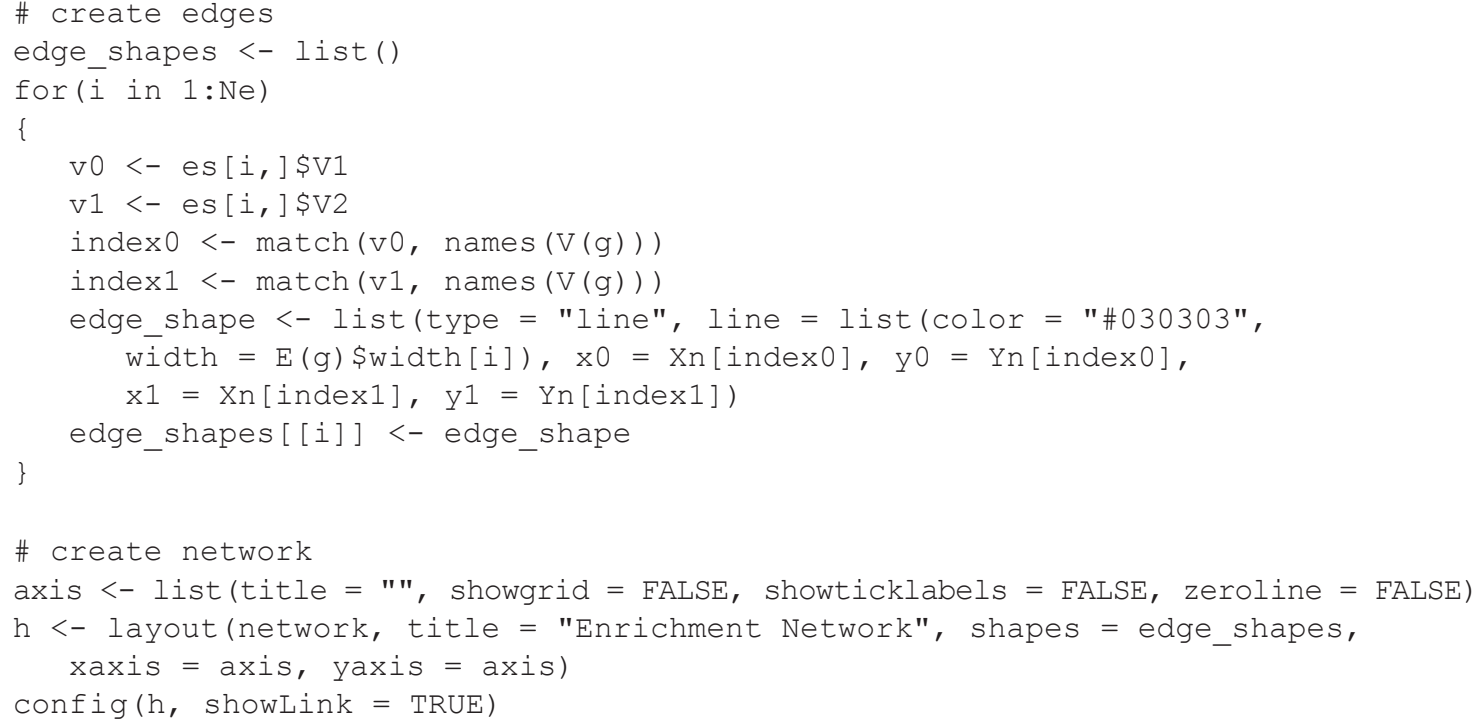

In Figure 3, the network visualization of the functional enrichment analysis is displayed. Nodes indicate GO terms and KEGG pathways. The connection between two nodes depends on the proportion of overlapping genes of corresponding two categories ${ }^{19}$. The size of nodes is proportional to the number of genes in their categories. The more significant categories are, the less transparent their nodes are. This network may be useful to see the overview of the functional enrichment.

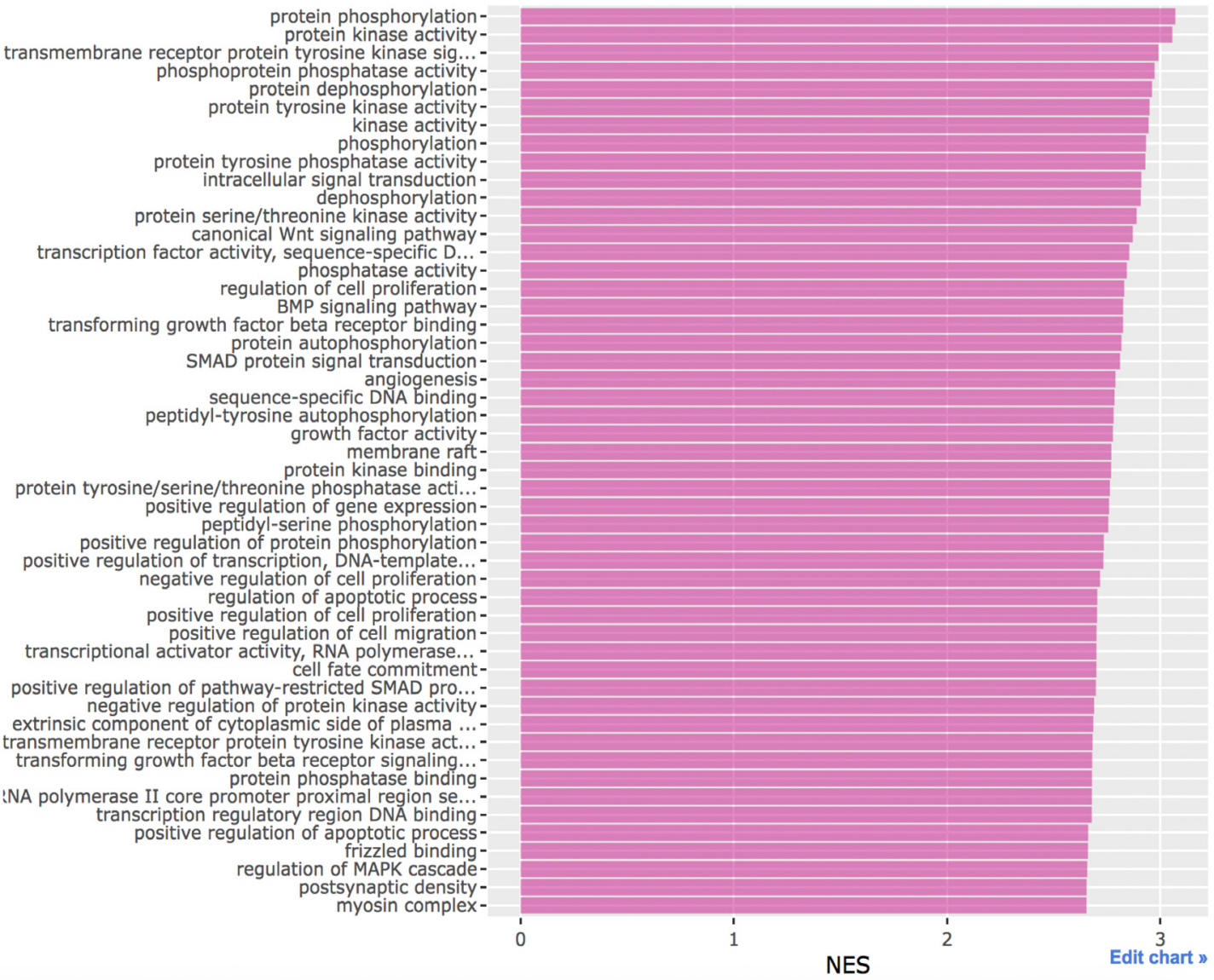

Figure 2. Top 50 categories from the GSEA with scaled scores of genes from the target, Ras signaling pathway. This figure was generated with Plotly (https://plot.ly/). 


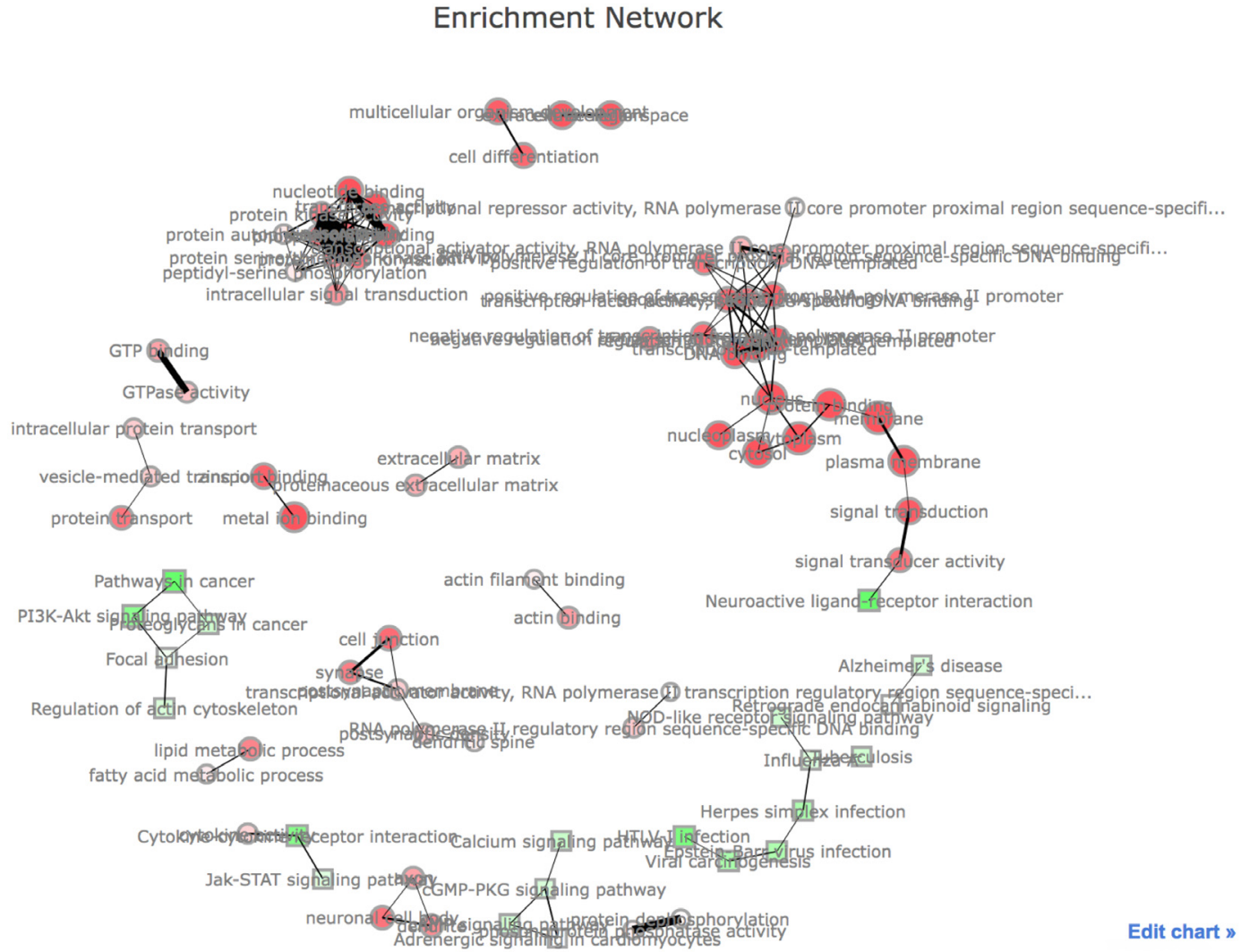

Figure 3. Network visualization with GO terms in red and KEGG pathways in green. This figure was generated with Plotly (https://plot.ly/).

\section{Discussion}

The proposed method is highly dependent on protein interaction networks. There are many prominent databases that include extensive information on protein interactions, and interactions are also reported in thousands of literature references. Therefore, the choice of data can be critical. Another issue is a technical problem. The one-class support vector machine can be sensitive to the choice of kernels and parameters. However, there is potential in inferring putative functions of proteins from PPIs, complementing conventional methods based on protein sequence analyses.

\section{Software availability}

The PPInfer package is available at: http://bioconductor.org/packages/PPInfer/

Source code is available at: https://github.com/Bioconductor-mirror/PPInfer

Archived source code as at the time of publication: https://doi.org/doi:10.5281/zenodo.1035128 20

License: Artistic-2.0

Competing interests

No competing interests were disclosed.

Grant information

This material is based upon work supported by the National Science Foundation/EPSCoR Grant Number IIA-1355423 and by the State of South Dakota.

The funders had no role in study design, data collection and analysis, decision to publish, or preparation of the manuscript. 
1. Chatr-Aryamontri A, Breitkreutz BJ, Heinicke S, et al: The BioGRID interaction database: 2013 update. Nucleic Acids Res. 2013; 41(Database issue): D816-D823.

PubMed Abstract | Publisher Full Text | Free Full Text

2. Szklarczyk D, Franceschini A, Wyder S, et al.: STRING v10: proteinprotein interaction networks, integrated over the tree of life. Nucleic Acids Res. 2015; 41(Database issue): D447-52. PubMed Abstract | Publisher Full Text | Free Full Text

3. Fellenberg $\mathrm{M}$, Albermann $\mathrm{K}$, Zollner $\mathrm{A}$, et al.: Integrative analysis of protein interaction data. Proc Int Conf Intell Syst Mol Biol. 2000; 8: 152-161.

PubMed Abstract

4. Schwikowski B, Uetz P, Fields S: A network of protein-protein interactions in yeast. Nat Biotechnol. 2000; 18(12): 1257-1261. PubMed Abstract | Publisher Full Text

5. Deng M, Zhang K, Mehta S, et al: Prediction of protein function using protein-protein interaction data. J Comput Biol. 2003; 10(6): 947-960.

PubMed Abstract | Publisher Full Text

6. Deng M, Chen T, Sun F: An integrated probabilistic model for functional prediction of proteins. J Comput Biol. 2004; 11(2-3): 463-475.

PubMed Abstract | Publisher Full Text

7. Joshi T, Chen Y, Becker JM, et al.: Genome-scale gene function prediction using multiple sources of high-throughput data in yeast Saccharomyces cerevisiae. OMICS. 2004; 8(4): 322-333. PubMed Abstract | Publisher Full Text

8. Peng W, Wang J, Cai J, et al.: Improving protein function prediction using domain and protein complexes in PPI networks. BMC Syst Biol. 2014; 8(1): 35

PubMed Abstract | Publisher Full Text | Free Full Text

9. Vapnik V: The nature of statistical learning theory. Springer-Verlag. 995.

Reference Source

10. Cover TM: Geometrical and statistical properties of systems of linear inequalities with applications in pattern recognition. IEEE
Transactions on Electronic Computers. 1965; EC-14(3): 326-334. Publisher Full Text

11. Kondor R, Lafferty J: Diffusion kernels on graphs and other discrete structures. In Proceedings of the 19th International Conference on Machine Learning.2002; 315-322. Reference Source

12. Cook DJ, Holder LB (Eds.): Mining graph data. John Wiley \& Sons. 2006.

Publisher Full Text

13. Smola AJ, Kondor R: Kernels and regularization on graphs. In Learning theory and kernel machines. Springer. 2003; 144-158. Publisher Full Text

14. Samatova NF, Hendrix W, Jenkins J, et al.(Eds.): Practical graph mining with R. CRC Press. 2013. Reference Source

15. Kolaczyk ED, Csardi G: Statistical analysis of network data with $\mathbf{R}$. Springer. 2014. Publisher Full Text

16. Scholkopf B, Burges CJ, Smola AJ: Advances in kernel methods: support vector learning. 1999. MIT press. Reference Source

17. Ma Y, Guo G: Support vector machines applications. Springer 2014. Publisher Full Text

18. Senay SD, Worner SP, Ikeda T: Novel three-step pseudo-absence selection technique for improved species distribution modelling. PLoS One. 2013; 8(8): e71218. PubMed Abstract | Publisher Full Text | Free Full Text

19. Yu G, Wang LG, Yan GR, et al:: DOSE: an R/Bioconductor package for disease ontology semantic and enrichment analysis Bioinformatics. 2014; 31(4): 608-609. PubMed Abstract | Publisher Full Text

20. Jung D, Ge X: PPInfer: a Bioconductor package for inferring functionally related proteins using protein interaction networks. Zenodo. 2017. Data Source 


\section{Open Peer Review}

\section{Current Peer Review Status: ?}

\section{Version 1}

Reviewer Report 22 November 2017

https://doi.org/10.5256/f1000research.14038.r27695

(c) 2017 Wu C et al. This is an open access peer review report distributed under the terms of the Creative Commons Attribution License, which permits unrestricted use, distribution, and reproduction in any medium, provided the original work is properly cited.

\section{Cathy H. Wu}

Center for Bioinformatics \& Computational Biology (CBCB), University of Delaware, Newark, DE, USA

\section{Karen E. Ross}

Department of Biochemistry and Molecular \& Cellular Biology, Georgetown University Medical Center, Washington, DC, USA

The article describes a new R package, PPInfer, for inferring functions of proteins based on their connectivity in a PPI network. Discovery of new functions for proteins is an important problem and the method as described is an interesting and theoretically sound approach.

The authors provide detailed example code for using PPInfer. Unfortunately, when trying to reproduce the use case, there was an error in the first step:

\section{> K.10090 <- readRDS("K10090.rds")}

Error in gzfile(file, "rb") : cannot open the connection

In addition: Warning message:

In gzfile(file, "rb") :

cannot open compressed file 'K10090.rds', probable reason 'No such file or directory'

Therefore, we couldn't test the rest of the use case.

Also, in general, the use case would benefit from more text explanation so that readers understand the purpose of the tool and how to interpret the results. Specifically:

1. What is the meaning of the gene ratio on the $x$-axis in Figure 1?

2. In Figure 3, are the nodes enriched KEGG pathways and GO terms as determined by GSEA? What was the threshold for deciding which nodes should appear in the graph? What is the biological message of this graph?

3. A discussion of the functionally related proteins themselves (not just the enriched 
terms/pathways) would be helpful. How do these proteins functionally interact with the ras pathway proteins in the STRING network? Perhaps you could discuss the STRING confidence scores or types of evidence for these interactions.

4. It would be helpful to have more discussion of exactly what we learn from this approach. Can we infer that the proteins identified in the use case might be involved in the Ras pathway? It seems like based on the GO/KEGG annotation for the proteins, we could already have assumed they had something to do with signal transduction even without knowing that they are closely associated with Ras pathway proteins. Are there any examples of proteins whose association with the Ras pathway was surprising? Are there any proteins about which little is known or whose GO/KEGG annotation does not indiciate that they would be associated with the Ras pathway?

Please note that for questions 3,4 , and 5 on the referee report form: we were unable to fully evaluate this as we were unable to run the software. We have therefore assigned the answer "no" to reflect this.

Is the rationale for developing the new software tool clearly explained?

Partly

Is the description of the software tool technically sound?

Yes

Are sufficient details of the code, methods and analysis (if applicable) provided to allow replication of the software development and its use by others?

No

Is sufficient information provided to allow interpretation of the expected output datasets and any results generated using the tool?

No

Are the conclusions about the tool and its performance adequately supported by the findings presented in the article?

No

Competing Interests: No competing interests were disclosed.

We confirm that we have read this submission and believe that we have an appropriate level of expertise to confirm that it is of an acceptable scientific standard, however we have significant reservations, as outlined above.

Author Response 01 Dec 2017

Dongmin Jung, Yonsei University, Seoul, South Korea

Thank you for taking the time to read and review our paper. In the revised version, we have improved the clarity of the figures and the text in several places, according to your 
suggestions.

Necessary files are available at https://zenodo.org/record/1066236. Please, download K10090.rds and install the current version of this package.

In Figure 1, The horizontal axis represents the proportion of our interesting genes in a certain functional category.

In Figure 3, the 200 most significant categories from the GSEA are used, with the cutoff of 0.05 and 0.2 for p-values and edges, respectively. For GO terms, the two largest subnetworks are involved in transcription and signal transduction. We can see that Ras is related to tumorigenesis via the PI3K-Akt pathway from KEGG.

For the discussion of the functionally related proteins themselves, two figures are added. One is the heatmap for the relationship between top proteins and pathways in Figure 4. The other is visualization of PPI among proteins in Figure 5. Please, see the text for further discussion.

Competing Interests: No competing interests were disclosed.

The benefits of publishing with F1000Research:

- Your article is published within days, with no editorial bias

- You can publish traditional articles, null/negative results, case reports, data notes and more

- The peer review process is transparent and collaborative

- Your article is indexed in PubMed after passing peer review

- Dedicated customer support at every stage

For pre-submission enquiries, contact research@f1000.com 